\title{
Improving the efficiency of polymer solar cells by incorporating gold nanoparticles into all polymer layers
}

\author{
Feng-Xian Xie, Wallace C. H. Choy, ${ }^{\text {a) }}$ Charlie C. D. Wang, Wei E. I. Sha, \\ and Dixon D. S. Fung \\ Department of Electrical and Electronic Engineering, The University of Hong Kong, Pokfulam Road, \\ Hong Kong, China
}

(Received 23 August 2011; accepted 16 September 2011; published online 11 October 2011)

\begin{abstract}
We demonstrate efficiency improvement in polymer solar cells (PSCs) by $\sim 22 \%$ through incorporating Au nanoparticles (NPs) into all polymer layers. Au NPs are found to have distinct mechanisms in improving device performance when incorporated in different polymer layers. Au NPs in poly-(3,4-ethylenedioxythiophene):poly(styrenesulfonate) mainly contribute to better hole collection while Au NPs in active layer contributes to the enhanced optical absorption and more balanced charge-transport. Our theoretical result shows that the absorption enhancement at the active layer is attributed to plasmon resonances with strong near-field distributions penetrated into absorption polymers. These findings can be applied to design high-efficiency metallic NPs-incorporated PSCs. C 2011 American Institute of Physics. [doi:10.1063/1.3650707]
\end{abstract}

Polymer solar cells (PSCs) have been a highly interesting field in recent years, as they have a strong potential to realize low cost solar cells which are highly portable and deployable due to their flexibility and light weight. ${ }^{1}$ Compared with inorganic solar cells, PSCs usually suffer from the insufficient light absorption due to the thin active layer restricted by the short exciton diffusion length and low carrier mobilities. ${ }^{2-4}$ To overcome these limits, metallic (e.g., Au, Ag) nanoparticles (NPs) have been incorporated into the polymer layers conveniently in solution processing. Although the power conversion efficiency (PCE) of PSCs has been shown to improve by incorporating metallic NPs in either the buffer layer such as poly-(3,4-ethylenedioxythiophene):poly(styrenesulfonate) (PEDOT:PSS) or the active layer, ${ }^{5-10}$ the understanding on the changes is still not quite clear.

In this work, monofunctional poly(ethylene glycol) (PEG)-capped Au NPs of sizes $18 \mathrm{~nm}$ and $35 \mathrm{~nm}$ are doped in the PEDOT:PSS and poly(3-hexylthiophene) (P3HT):phenylC61-butyric acid methyl ester (PCBM) layers, respectively, leading to an improvement of PCE by $\sim 22 \%$ compared to the optimized control device. We first identify the impact of NPs in each polymer layer on PSC characteristics by doping $\mathrm{Au}$ NPs in either the PEDOT:PSS or P3HT:PCBM layer. Then, we investigate the impact of Au NPs when incorporated in all polymer layers. We demonstrate that the accumulated benefits of incorporating Au NPs in all organic layers of PSCs can achieve larger improvements in PSC performances.

The basic PSC structure in this work is ITO/PEDOT:PSS/ P3HT:PCBM/LiF (1 nm)/Al (100 nm). Device A has no NP doping, while devices B, C, and D have NPs doped into PEDOT:PSS, P3HT:PCBM, and both layers, respectively. For hole-only devices, $\mathrm{Au}(20 \mathrm{~nm}) / \mathrm{Al}(80 \mathrm{~nm})$ electrode was used in place of the $\mathrm{LiF} / \mathrm{Al}$ electrode. Detailed information of the optimized conditions for the control device A can be found elsewhere. ${ }^{11}$ The synthesis of Au NPs with PEG capping are

\footnotetext{
${ }^{\text {a) }}$ Author to whom correspondence should be addressed. Electronic mail: chchoy@eee.hku.hk.
}

described our previous report. ${ }^{10}$ The amount of Au NPs doped into the organic layer $(0.32 \mathrm{wt}$. \% in PEDOT:PSS and $1.2 \mathrm{wt}$. $\%$ in P3HT:PCBM) had been optimized for the best PCE. Device characterization methods including current density $(J)$ voltage $(V)$ characteristics, optical absorption, atomic force microscopy, (AFM) and scanning electron microscope (SEM) were conducted as stated elsewhere. ${ }^{10-12}$ The volume integral equation (VIE) method, which is a rigorous solution to Maxwell's equations, was adopted to study the optical absorption of PSCs. The coupling between multiple NPs and the interaction between NPs and multilayered device structure were fully considered. $^{13}$

$J-V$ characteristics of the four PSC device structures with Au NPs incorporated into different organic layers are shown in Fig. 1(a), and the photovoltaic parameters are listed in Table I. We observe that the incorporation of Au NPs into either the PEDOT:PSS layer (device B) or the active layer (device C) improves PCE from $3.16 \%$ (control device A) to $3.61 \%$ and $3.44 \%$, respectively. Interestingly, the simultaneous incorporation of Au NPs into both layers results in a further improvement of average PCE to $3.85 \%$. In all cases, the increases in PCE are results of improvements in short-circuit current density $\left(J_{S C}\right)$ and fill factor $(F F)$, while the open-circuit voltage $\left(V_{O C}\right)$ is unchanged. Notably, when incorporating Au NPs into both PEDOT:PSS and active layer simultaneously, the series resistance $\left(R_{S}\right)$ reduces obviously from $3.05 \Omega \mathrm{cm}^{2}$ (device A) to $1.93 \Omega \mathrm{cm}^{2}$ (device D), contributing to a remarkable increase of $F F$ from $61.92 \%$ to $65.00 \%$.

The effect of NPs doped in a single layer is first studied. For the case of Au NPs doped into the PEDOT:PSS layer only (device B), a study of the physics has been conducted previously. ${ }^{10}$ In good agreement with our previous results, the absorption spectrum of the active layer does not show any clear change from the control device $\mathrm{A}$ as shown in Fig. 1(b). This can be explained by our finding that the strong near field around Au NPs due to the localized surface plasmon resonance (LSPR) distributes laterally along the PEDOT:PSS layer instead of vertically penetrating into the 

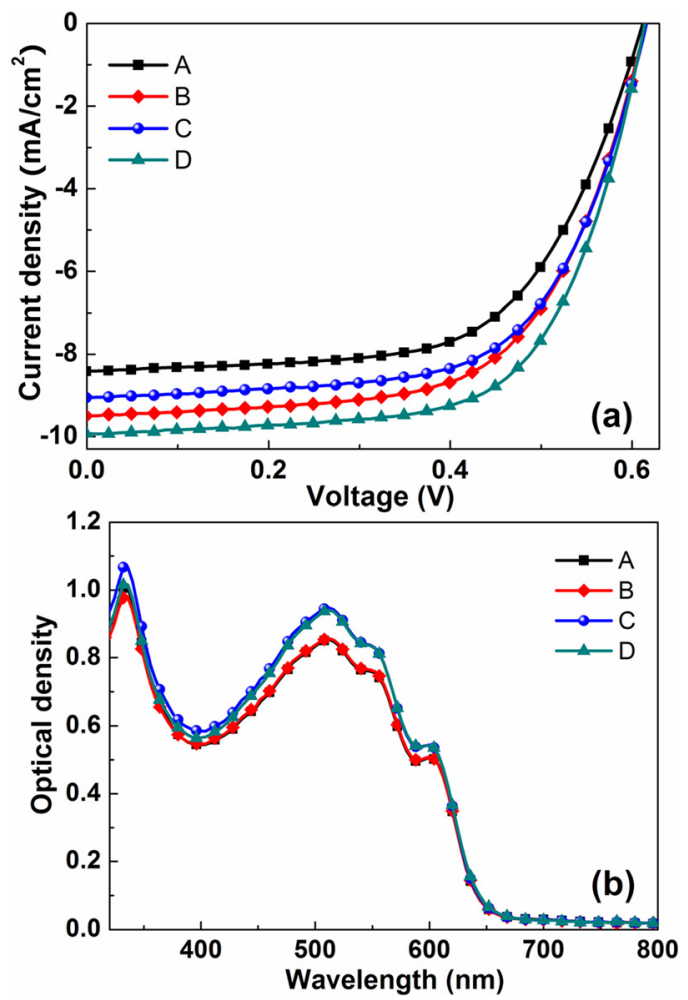

FIG. 1. (Color online) (a) $J-V$ characteristics of the PSCs with NPs incorporated into different layers under AM $1.5 \mathrm{G}$ illumination at $100 \mathrm{~mW} / \mathrm{cm}^{2}$. (b) Absorbance of the active layer for different NP doping structures. (device A: No NPs; B: NPs in PEDOT:PSS only; C: NPs in P3HT:PCBM only; and D: NPs in both P3HT:PCBM and PEDOT:PSS).

active layer. ${ }^{10}$ Meanwhile, the hole mobilities of devices A and $\mathrm{B}$ derived from the hole-only devices show negligible changes (Supporting information: Fig. S1), ${ }^{17}$ indicating that charge transport in the bulk of the active layer is unaffected by NPs incorporated in PEDOT:PSS. The surface morphology of the PEDOT:PSS + Au NPs layer (Supporting information: Fig. S2) ${ }^{17}$ shows an obvious increase in surface roughness with the root mean square (RMS) roughness increasing from $0.97 \mathrm{~nm}$ to $1.55 \mathrm{~nm}$. Therefore, the incorporation of Au NPs only in PEDOT:PSS increases the interfacial contact area between the P3HT:PCBM and PEDOT:PSS, allowing more efficient hole collection at the anode and hence improves $J_{S C}$ and $F F .^{14,15}$ Furthermore, from resistive devices of structure ITO/PEDOT:PSS (with or without $\mathrm{Au} \mathrm{NPs}$ /Al, it is found that the resistance of PEDOT:PSS reduces upon addition of NPs (Supporting information: Fig. S3 (Ref. 17)). Both the increased interfacial contact area and conductivity of PEDOT:PSS contribute to

TABLE I. Photovoltaic parameters of the PSCs with NPs incorporated in different layers under AM $1.5 \mathrm{G}$ illumination at $100 \mathrm{~mW} / \mathrm{cm}^{2} . R_{S}$ is derived from the slope of the current-voltage $(J-V)$ curves under dark at $2 \mathrm{~V}$. (device A: control device without NPs; B: NPs in PEDOT:PSS only; C: NPs in P3HT:PCBM only; D: NPs in both P3HT:PCBM and PEDOT:PSS).

\begin{tabular}{lccccc}
\hline \hline Device & $V_{O C}(\mathrm{~V})$ & $J_{S C}\left(\mathrm{~mA} / \mathrm{cm}^{2}\right)$ & $F F(\%)$ & $\operatorname{PCE}(\%)$ & $R_{S}\left(\Omega \mathrm{cm}^{2}\right)$ \\
\hline $\mathrm{A}$ & $0.61 \pm 0.00$ & $8.35 \pm 0.09$ & $61.92 \pm 0.33$ & $3.16 \pm 0.04$ & $3.05 \pm 0.03$ \\
$\mathrm{~B}$ & $0.61 \pm 0.01$ & $9.41 \pm 0.28$ & $62.52 \pm 0.66$ & $3.61 \pm 0.08$ & $2.11 \pm 0.06$ \\
$\mathrm{C}$ & $0.61 \pm 0.00$ & $8.85 \pm 0.27$ & $63.56 \pm 0.53$ & $3.41 \pm 0.11$ & $2.92 \pm 0.13$ \\
$\mathrm{D}$ & $0.61 \pm 0.01$ & $9.74 \pm 0.57$ & $65.00 \pm 1.02$ & $3.85 \pm 0.20$ & $1.93 \pm 0.04$ \\
\hline \hline
\end{tabular}

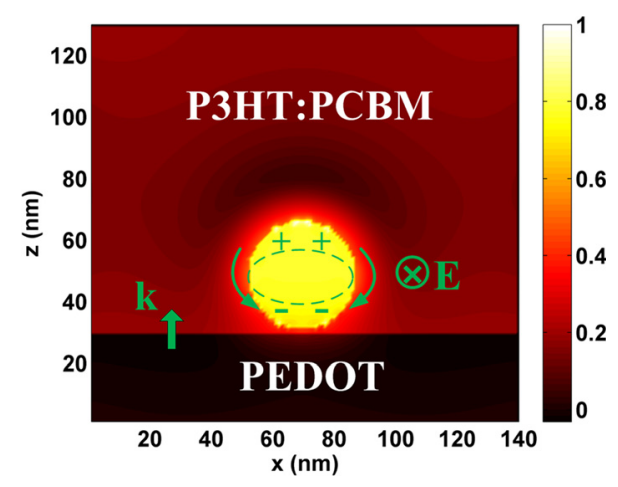

FIG. 2. (Color online) The schematic pattern for the plasmon resonance and charge distribution of a Au NP, as well as the near-field distributions for the vertically incident light with TE polarization in P3HT:PCBM.

the reduction of the series resistance of PSCs from $3.05 \Omega$ $\mathrm{cm}^{2}$ to $2.11 \Omega \mathrm{cm}^{2}$ and improvement of $F F$ and PCE.

Although electrical effects dominantly address the performance improvement when incorporating Au NPs only in PEDOT:PSS, the mechanism for PCE improvement is found to be different when NPs are incorporated into the active layer. As shown in Fig. 1(b), when NPs are incorporated into the active layer only (device $\mathrm{C}$ ), absorption of the active layer increases over a wide wavelength range. From our theoretical studies, we find that the absorption enhancement can be explained by LSPRs in the Au NPs excited by the TE polarized light. The dipoles generated in the Au NPs and the strong near field by LSPRs by Au NPs in the active layer are shown in Fig. 2. It can be observed that the strong near field distributes into the active layer and directly enhances the light absorption by the blended polymers of the active layer. Together the scattering effects of Au NPs coupled to waveguide mode, $J_{S C}$ improves. Regarding electrical properties, hole-only devices indicate that hole mobility increases from $8.94 \times 10^{-4} \mathrm{~cm}^{2} / \mathrm{V}$ s to $1.19 \times 10^{-3} \mathrm{~cm}^{2} / \mathrm{V}$ s. Studies have shown that in P3HT:PCBM, electron mobility is higher than hole mobility and this carrier imbalance is detrimental to photovoltaic performance. ${ }^{16}$ The increase in hole mobility allows more balanced charge transport in the active layer, thus improving the $J_{S C}$ and $F F$ of the device.

When Au NPs are doped into both PEDOT:PSS and P3HT:PCBM, PCE further increases to $3.85 \%$. From holeonly devices, the hole mobility of P3HT:PCBM is determined to be $\sim 1.21 \times 10^{-3} \mathrm{~cm}^{2} / \mathrm{V} \mathrm{s}$, which is similar to the case when $\mathrm{NP}$ is doped in the active layer only $\left(1.19 \times 10^{-3} \mathrm{~cm}^{2} / \mathrm{V} \mathrm{s}\right)$. From the cross section SEM images as shown in Fig. 3, Au NPs doped in the active layer are mostly located at the bottom of P3HT:PCBM (near to the interface with PEDOT:PSS), while the Au NPs doped to PEDOT:PSS are well embedded in the PEDOT:PSS layer. However, despite the fact that the active layer is adjacent to the PEDOT:PSS layer, the highly similar absorption spectra of devices $\mathrm{C}$ (Au NPs in P3HT:PCBM only) and D (Au NPs in both P3HT:PCBM and PEDOT:PSS) in Fig. 1(b) show that there is no clear interaction between the Au NPs in the active layer and those in the PEDOT:PSS layer. From the principle of optics, the absence of clear couplings between Au NPs in PEDOT and Au NPs in P3HT:PCBM is reasonable because the polarization direction of the electric field of vertically incident light is parallel to each device layer. Furthermore, we have shown that the 


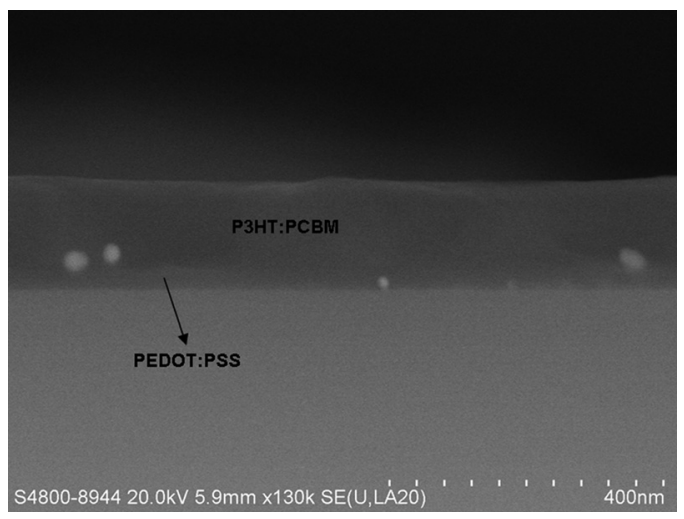

FIG. 3. Representative cross section SEM image of the film structure PEDOT:PSS + Au NPs/P3HT:PCBM + Au NPs.

improvement mechanisms when incorporating NPs in only PEDOT:PSS or P3HT:PCBM mainly originate from interfacial or bulk effects, respectively. As interfacial and bulk effects are two separate effects and optical coupling between NPs is not observed, we expect that the improvement of PCE from $3.16 \%$ to $3.85 \%$ is the accumulated improvements of addition of NPs into the individual layers.

In conclusion, we have demonstrated $\sim 22 \%$ improvement in efficiency by incorporating Au NPs into all organic layers in the PSCs. The improvement is attributed to the accumulated enhancements in device performance due to addition of NPs into individual layers, while coupling between NPs in different layers is not observed. Our study shows that the advantages of incorporating NPs in individual layers can be utilized together to achieve larger increases of PSC performance and these findings can be applied to the design of high efficiency NP-incorporated PSCs in the future.
This work is financially supported by UGC grants (\#10400897 and \#10401466) of the University of Hong Kong, the General Research Fund (HKU\#712010) from the Research Grants Council of Hong Kong Special Administrative Region, China, and Jiawei SolarChina, Co. Ltd.

${ }^{1}$ G. Dennler, M. C. Scharber, and C. J. Brabec, Adv. Mater. 21, 1323 (2009).

${ }^{2}$ D. Wöhrle and D. Meissner, Adv. Mater. 3, 129 (1991).

${ }^{3}$ V. Shrotriya, E. H.-E. Wu, G. Li, Y. Yao, and Y. Yang, Appl. Phys. Lett. 88, 064104 (2006).

${ }^{4}$ P. W. M. Blom, V. D. Mihailetchi, L. J. A. Koster, and D. E. Markov, Adv. Mater. 19, 1551 (2007).

${ }^{5}$ F.-C. Chen, J.-L. Wu, C.-L. Lee, Y. Hong, C.-H. Kuo, and M. H. Huang, Appl. Phys. Lett. 95, 013305 (2009).

${ }^{6}$ J.-L. Wu, F.-C. Chen, Y.-S. Hsiao, F.-C. Chien, P. Chen, C.-H. Kuo, M. H. Huang, and C.-S. Hsu, ACS Nano 5, 959 (2011).

${ }^{7}$ K. Kim and D. L. Carroll, Appl. Phys. Lett. 87, 203113 (2005).

${ }^{8}$ D. H. Wang, D. Y. Kim, K. W. Choi, J. H. Seo, S. H. Im, J. H. Park, O. O. Park, and A. J. Heeger, Angew. Chem., Int. Ed. 50, 5519 (2011).

${ }^{9}$ W. E. I. Sha, W. C. H. Choy, and W. C. Chew, Opt. Lett. 36, 478 (2011).

${ }^{10}$ D. D. S. Fung, L.-F. Qiao, W. C. H. Choy, C.-D. Wang, W. E. I. Sha, F.-X. Xie, and S. He, J. Mater. Chem. (2011).

${ }^{11}$ C.-D. Wang and W. C. H. Choy, Sol. Energy Mater. Sol. Cells 95, 904 (2011).

${ }^{12}$ F.-X. Xie, W. C. H. Choy, X. Zhu, X. Li, Z. Li, and C. Liang, Appl. Phys. Lett. 98, 243302 (2011).

${ }^{13}$ W. E. I. Sha, W. C. H. Choy, Y. P. Chen, and W. C. Chew, Opt. Express 19, 15908 (2011).

${ }^{14}$ M.-H. Hsu, P. Yu, J.-H. Huang, C.-H. Chang, C.-W. Wu, Y.-C. Cheng, and C.-W. Chu, Appl. Phys. Lett. 98, 073308 (2011).

${ }^{15}$ B. Peng, X. Guo, C. Cui, Y. Zou, C. Pan, and Y. Li, Appl. Phys. Lett. 98, 243308 (2011).

${ }^{16}$ G. Li, V. Shrotriya, Y. Yao, J. Huang, and Y. Yang, J. Mater. Chem. 17, 3126 (2007).

${ }^{17}$ See supplementary material at http://dx.doi.org/10.1063/1.3650707 for showing (1) the J-V characteristics of hole-only devices with NPs incorporated different region of the devices and (2) the change of surface morphology of PEDOT:PSS due to the incorporation of the NPs by using AFM. 